\section{Amiodarone-induced bone marrow granulomas: an unusual cause of reversible pancytopenia}

\author{
Andrew J. Erie, ${ }^{1}$ Rebecca F. McClure, ${ }^{2}$ \\ Alexandra P. Wolanskyj ${ }^{3}$ \\ 1 Mayo Medical School, 2Department \\ of Laboratory Medicine and Pathology, \\ ${ }^{3}$ Department of Internal Medicine, \\ Division of Hematology, Mayo Cinic, \\ Rochester, MN, USA
}

\begin{abstract}
Bone marrow infiltration by granulomas rarely presents with cytopenias and is usually a result of atypical infections, lymphomas, or sarcoidosis. Drugs are also an important but often overlooked causal agent of bone marrow granulomas. Although rare, amiodarone has been associated with bone marrow granuloma formation. This case report describes a 73year-old male who presented with pancytopenia during a preoperative evaluation. Amiodarone therapy was suspected to be the causal agent after diagnostic evaluation and exclusion of other causes. After cessation of amiodarone, the patient's pancytopenia gradually resolved over a period of several months. Our report illustrates an often overlooked yet important cause of reversible pancytopenia owing to suspected amiodarone-induced bone marrow granuloma formation, and guides clinicians in an expected timeline for blood count improvement after cessation of this drug.
\end{abstract}

\section{Introduction}

Multifocal bone marrow granulomas result in pancytopenia by disrupting normal hematopoiesis. Granulomas form by an aggregation of activated macrophages that undergo a morphological transformation into epithelial-like cells. Granulomas may result from a chronic inflammatory response incited by persistent or non-degradable antigens. ${ }^{1}$ Identification of bone marrow granulomas is significant because they are usually a result of a limited number of conditions including malignant lymphomas, sarcoidosis, and atypical infections. Additional rarer causes include autoimmune disorders such as rheumatoid arthritis, systemic lupus erythematosus, and other malignancies. ${ }^{2-5}$ A variety of drugs have been reported to cause bone marrow granulomas, including procainamide, sulfonamide, and ibuprofen. ${ }^{6}$ More recently, amiodarone has been implicated as a potential cause of bone marrow granulomas and variable cytopenias, but rarely pancytopenia. ${ }^{7}$

\section{Case Report}

A 73-year-old man with severely symptomatic ischemic and valvular cardiomyopathy was found to have moderately severe pancytopenia (white blood cells, $2.4 \times 10^{9} / \mathrm{L}$; absolute neutrophils, $1.39 \times 10^{9} / \mathrm{L}$; platelets, $65 \times 10^{9} / \mathrm{L}$; hemoglobin, $9.8 \mathrm{~g} / \mathrm{dL}$ with a normal MCV) during a preoperative evaluation, prior to planned tricuspid valve replacement surgery. The physical examination was consistent with tricuspid valve regurgitation, but notably splenomegaly and lymphadenopathy were absent. A peripheral blood smear confirmed thrombocytopenia but was otherwise unremarkable. A bone marrow biopsy was performed to clarify the etiology of the pancytopenia prior to surgical intervention. The bone marrow biopsy revealed normal trilineage hematopoeisis. Cytogenetics and flow cytometry were likewise normal. Surprisingly, multiple small non-caseating granulomas were present (Figure 1). Special stains, cultures, and serological testing for associated infectious causes of bone marrow granulomas, including mycobacterial, fungal (Aspergillus, Cryptococcus, Histoplasmosis, Sporothrix, Coccidioides, Blastomyces), aerobic and anaerobic bacteria (particularly Brucellosis and Q-fever), were negative. There was no clinical evidence for a viral infection such as HIV, Epstein-Barr virus, or cytomegalovirus, nor for an autoimmune disorder such as rheumatoid arthritis or systemic lupus erythematosus. Inflammatory markers including sedimentation rate and C-reactive protein were both normal. Computed tomography of the chest and angiotensin converting enzyme levels were also unremarkable.

A review of our patient's history revealed that amiodarone had been started several years earlier for ventricular tachyarrhythmias, and mild cytopenias had been noted several months after initiation of this drug. Careful review of all other medications failed to reveal an obvious temporal association with his cytopenias. As such, amiodarone was thought to be a potential causal agent for the bone marrow granuloma formation and pancytopenia, and was discontinued.

Within six weeks after stopping amiodarone, with no other medication changes, our patient's blood counts improved significantly. He then underwent successful tricuspid valve replacement without postoperative complications related to cytopenias, despite the initiation of warfarin anticoagulation. At the four month follow-up, our patient's complete blood count returned to near normal levels (white
Correspondence: Alexandra P. Wolanskyj, Assistant Professor of Medicine, Division of Hematology, Mayo Clinic, $2001^{\text {st }}$ Street SW, Rochester, MN 55905, USA.

E-mail: wolanskyj.alexandra@mayo.edu

Key words: pancytopenia, granulomas, amiodarone.

Contributions: AE compiled the patient data, did the literature review, and wrote the manuscript; RMcC provided the pathology images; AW evaluated the patient and was involved in all aspects of patient care, chart review, and manuscript preparation.

Acknowledgments: written informed consent was obtained from the patient for publication of this case report and any accompanying images.

Conflict of interest: the authors report no conflicts of interest.

Received for publication: 17 July 2010 . Revision received: 6 September 2010. Accepted for publication: 16 September 2010.

This work is licensed under a Creative Commons Attribution 3.0 License (by-nc 3.0).

(C) Copyright A.J. Erie et al., 2010

Licensee PAGEPress, Italy

Hematology Reports 2010; 2:e6

doi:10.4081/hr.2010.e6

blood cells, $4.2 \times 10^{9} / \mathrm{L}$; absolute neutrophils, $2.36 \times 10^{9} / \mathrm{L}$; platelets, $106 \times 10^{9} / \mathrm{L}$; hemoglobin, $12.8 \mathrm{~g} / \mathrm{dL}$; Table 1) and he remained asymptomatic at the last outpatient follow-up.

\section{Discussion}

Amiodarone is a commonly prescribed class III antiarrhythmic medication that increasingly has been recognized in sporadic case reports as a potential cause of bone marrow granulomas. ${ }^{7-13}$ In several reports, amiodarone was not stopped because the treating physicians determined that the cardiac benefits outweighed the potential hematologic risks. ${ }^{9,11,12}$ Boutros et al. ${ }^{8}$ and Moran and Manoharan ${ }^{10}$ observed an improvement in cytopenias that paralleled a partial or complete resolution of bone marrow granulomas by three to eight months after cessation of amiodarone. Mohamed et al. ${ }^{9}$ reported a patient with leukopenia and thrombocytopenia that improved by six months after discontinuation of amiodarone. Similarly, our patient's hemoglobin, white blood cells, and platelets began to increase at six weeks after cessation of this drug and all three cell lines reached near nor- 
Table 1. Timeline of complete blood count recovery following cessation of amiodarone.

\begin{tabular}{lccc} 
Weeks after cessation & $\mathbf{H g}^{*}(\mathrm{~g} / \mathrm{dL})$ & WBC $^{\circ}\left(\mathbf{1} \mathbf{1 0}^{9 / \mathrm{L}}\right)$ & Platelets $\left(\times 10^{9 / L}\right)$ \\
0 weeks & 9.8 & 2.5 & 69 \\
6 weeks & 10.8 & 3.0 & 86 \\
\hline 11 weeks & 11.6 & 3.3 & 97 \\
16 weeks & 12.8 & 4.2 & 106 \\
\hline
\end{tabular}

${ }^{*} \mathrm{Hg}$, hemoglobin; ${ }^{\circ} \mathrm{WBC}$, white blood cell.

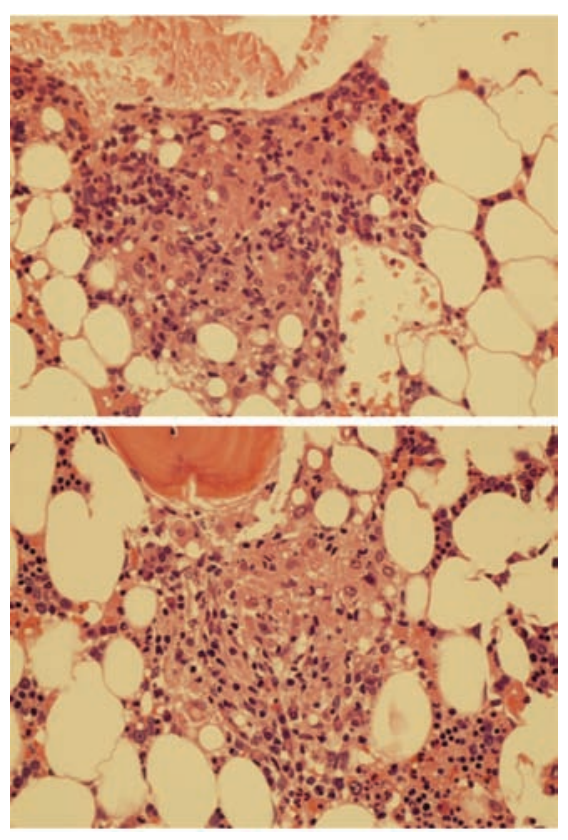

Figure 1. Non-caseating granulomas on bone marrow biopsy (hematoxylin and eosin stain; magnification: $400 \mathrm{X}$ ).

mal levels by four months. Close follow-up and regular blood count monitoring allowed us to define the expected temporal association between amiodarone cessation and blood count recovery (Table 1). The gradual improvement observed in both our patient's blood counts and previous published reports is consistent with the exceptionally long half-life of amiodarone. ${ }^{14}$ Although a repeat bone marrow biopsy after its discontinuation would offer definitive proof, this was not done as it was not clinically indicated. Similarly re-exposure to the drug to illicit a relapse was not ethically justifiable. Nevertheless, our patient's clinical evolution suggests an association between amiodarone, bone marrow granuloma formation, and resultant pancytopenia.

\section{Conclusions}

Our case implicates amiodarone as a cause of bone marrow granulomas with associated pancytopenia, and helps guide clinicians in an expected time course for improvement after drug cessation. Although reversible, the long half-life of amiodarone did result in a protracted duration of our patient's pancytopenia. Early recognition and diagnosis might limit the severity of the associated cytopenias, and mitigate the subsequent bleeding and infectious complications, and potentially reduce the need for transfusions. Amiodarone should be included in the differential diagnosis of patients who present with bone marrow granulomas and secondary cytopenias, and pancytopenia should be added as an additional reversible side effect of this commonly prescribed antiarrhythmic drug.

\section{References}

1. Kumar V, Abbas A, Fausto N. (eds) Robbins and Cotran Pathologic Basis of Disease. 7th ed. Oxford, Elsevier Saunders, 2005.

2. Bhargava V, Farhi D. Bone marrow granulomas: Clinicopathologic findings in 72 cases and review of the literature. Hematol Pathol 1988;2:43-50.
3. White RM, Johnston CL Jr. Granulomatous bone marrow disease in Virginia: Study of 50 cases. Va Med 1985;112:316-9.

4. Bodem CR, Hamory BH, Taylor HM, Kleopfer L. Granulomatous bone marrow disease - A review of the literature and clinicopathologic analysis of 58 cases. Medicine (Baltimore) 1983;62:372-83.

5. Vilalta-Castel E, Valdes-Sanchez MD, Guerra-Vales JM, et al. Significance of granulomas in bone marrow: A study of 40 cases. Eur J Haematol 1988;41:12-6.

6. Eid A, Carion W, Nystrom JS. Differential diagnoses of bone marrow granuloma. West J Med 1996;164:510-5.

7. Bilello SJ, Bao F, Veillon DM, Muldoon R, Cotelingam JD. Pathology case of the month. Elderly man with pancytopenia. Bone marrow granulomas secondary to amiodarone. J La State Med Soc 2006;158: 10-2.

8. Boutros NY, Dilly S, Bevan DH. Amiodarone-induced bone marrow granulomas. Clin Lab Haematol 2000;22:167-70.

9. Mohamed T, Sanjay R, Sycheva T, Aish L, Schneider D, Oo TH. Amiodarone-associated bone marrow granulomas: a report of 2 cases and review of the literature. Int $\mathrm{J}$ Hematol 2007;85:101-4.

10. Moran SK, Manoharan A. Amiodaroneinduced bone marrow granulomas. Pathology 2002;34:267-9.

11. Mukhopadhyay S, Mukhopadhyay S, Abraham NZ Jr, Jones LA, Howard L, Gajra A. Unexplained bone marrow granulomas: is amiodarone the culprit? A report of 2 cases. Am J Hematol 2004;75:110-2.

12. Rosenbaum H, Ben-Arie Y, Azzam ZS, Krivoy N. Amiodarone-associated granuloma in bone marrow. Ann Pharmacother 1998;32:60-2.

13. Yamreudeewong W, McIntyre WW, Sun TJ, Ranelli PL. Bone marrow granulomas possibly associated with amiodarone. Pharmacotherapy 2000;20:855-9.

14. Doggrell S. Amiodarone - waxed and waned and waxed again. Expert Opin Pharmacother 2001;2:1877-90. 